Applied Physiology, Nutrition, and Metabolism

Canadian Science Publishing Physiologie appliquée, nutrition et métabolisme

\title{
Postprandial Walking Reduces Glucose Levels in Women with Gestational Diabetes Mellitus
}

\begin{tabular}{|r|l|}
\hline Journal: & Applied Physiology, Nutrition, and Metabolism \\
\hline Manuscript ID & apnm-2017-0494.R2 \\
\hline Manuscript Type: & Brief communication \\
\hline Complete List of Authors: & $\begin{array}{l}\text { Coe, Dawn; The University of Tennessee Knoxville, Department of } \\
\text { Kinesiology, Recreation, and Sport Studies } \\
\text { Conger, Scott; Boise State University } \\
\text { Kendrick, Jo; University of Tennessee } \\
\text { Howard, Bobby; University of Tennessee } \\
\text { Thompson, Dixie; University of Tennessee } \\
\text { Bassett, David; University of Tennessee, } \\
\text { White, Jennifer; University of Tennessee }\end{array}$ \\
\hline Keyword: & $\begin{array}{l}\text { pregnancy, gestational diabetes mellitus, Moderate Intensity, Continuous } \\
\text { Glucose Monitoring System, Walking }\end{array}$ \\
\hline Is the invited manuscript for \\
consideration in a Special \\
Issue? :
\end{tabular}




\title{
Postprandial Walking Reduces Glucose Levels in Women with Gestational Diabetes Mellitus
}

\author{
Dawn P. Coe, Scott A. Conger, Jo M. Kendrick, Bobby C. Howard, Dixie L. Thompson, David
}

R. Bassett, Jr., and Jennifer D. White

\section{Dawn P. Coe, Ph.D. (Corresponding Author)}

Department of Kinesiology, Recreation, and Sport Studies

University of Tennessee

1914 Andy Holt Avenue, 341 HPER

Knoxville, TN 37996-2700

865-974-0294 (Phone)

865-974-8981(Fax)

dcoe@utk.edu

Scott A. Conger, Ph.D.

Boise State University

Department of Kinesiology

910 University Drive

Boise, ID 83725

scottconger@boisestate.edu

Jo M. Kendrick, W.H.N.P.-B.C.

The University of Tennessee, Knoxville, Medical Center

Department of Obstetrics and Gynecology

1924 Alcoa Hwy Bldg A Ste 435

Knoxville, TN 37920

JKendrick@utmck.edu

Bobby C. Howard, M.D.

The University of Tennessee, Knoxville, Medical Center

Department of Obstetrics and Gynecology

1924 Alcoa Hwy Bldg A Ste 435

Knoxville, TN 37920

bhowar12@utmck.edu

Dixie L. Thompson, Ph.D.

The University of Tennessee, Knoxville

Department of Kinesiology, Recreation, and Sport Studies

111 Student Services Bldg.

dixielee@utk.edu 
45 David R. Bassett, Jr., Ph.D.

46 The University of Tennessee, Knoxville

47 Department of Kinesiology, Recreation, and Sport Studies

$48 \quad 1914$ Andy Holt Avenue, 328 HPER

49 Knoxville, TN 37996-2700

50 dbassett@utk.edu

51

52 Jennifer D. White M.S.

53 The University of Tennessee, Knoxville

54 Department of Kinesiology, Recreation, and Sport Studies

551914 Andy Holt Avenue, 309 HPER

56 Knoxville, TN 37996-2700

57 white.jennifer.d@gmail.com

58 


\section{Postprandial Walking Reduces Glucose Levels in Women with Gestational Diabetes Mellitus}

\section{Abstract}

63 The purpose of this study is to investigate blood glucose changes, as measured by a continuous

64 glucose monitoring system, that occur in women with gestational diabetes mellitus (GDM)

65 following an acute bout of moderate-intensity walking after consuming a high carbohydrate/low fat meal. This study found that moderate-intensity walking induced greater postprandial glucose

67 control compared to sedentary activity and it appears that moderate-intensity activity may be 68 used to reduce postprandial glucose levels in women with GDM.

69

70 Key Words: Pregnancy, Physical Activity, Continuous Glucose Monitoring System, Moderate 71 Intensity 


\section{Introduction}

Approximately 5-10\% of all pregnant women will develop GDM during their pregnancy (Chen et al., 2016, Public Health Agency of Canada, 2014). Treatment of GDM is critical to preventing complications for both the mother and the fetus. If satisfactory control of blood glucose cannot be achieved through dietary intervention, then insulin may be required. Physical activity is considered an adjunctive therapy, due to the insulin-like effect on glucose uptake in skeletal muscle (ACOG, 2015).

Physical activity interventions implemented during pregnancy have resulted in lower fasting glucose levels (Davenport et al., 2008; Jovanovic-Peterson et al., 1989). However, studies investigating blood glucose changes after an acute bout of activity have found only modest changes lasting 45 to 60 minutes post activity. This includes studies incorporating a variety of intensities (light to moderate), modes of activity (cycling, walking), and timing of activity (pre- and postprandial) (Avery \& Walker, 2001; Garcia-Patterson et al., 2001; Lesser et al., 1996). Postprandial glucose levels often represent the highest peak in maternal blood glucose, and may have a greater impact on insulin response and fetal glucose uptake compared to fasting glucose levels (Heine et al., 2004).

Physical activity recommendations during pregnancy include up to 30 minutes of moderate intensity activity on most or all days of the week (ACOG, 2015; Wolfe \& Davies, 2003, Colberg et al., 2016). Artal recommended a similar amount of moderate activity to take place approximately 30 minutes following a meal (2003). Observing the time course of changes in postprandial blood glucose levels following moderate-intensity walking may give greater insight into the physiological impact of postprandial physical activity in women with GDM. 
94 Therefore, the purpose of this study was to investigate blood glucose changes, as measured by

95 continuous glucose monitoring system (CGMS), that occurred following an acute bout of

96

97

\section{Materials and Methods \\ Participants}

Eight women with GDM $(29.2+5.1 \mathrm{y}, 1.7 \pm 0.6 \mathrm{~m}, 92.2 \pm 23.0 \mathrm{~kg})$ were recruited by a nurse practitioner from a high risk obstetric clinic. Participants were either in their second or third trimester (24-35 weeks), free of any contraindications to physical activity, as outlined in the ACOG guidelines (ACOG, 2015), and not using insulin. The Physical Activity Readiness Medical Examination for Pregnancy (PARmed-X for pregnancy) (Canadian Society for Exercise Physiology, 2002) was completed by a physician for all participants. This study was approved by the Institutional Review Board.

\section{Instruments}

\section{Medtronic iPro Recorder CGMS}

The $i$ Pro Recorder CGMS (Medtronic Inc.; Minneapolis, MN) has been validated and used in pregnant women with diabetes (Kerssen et al., 2004, 2005). The CGMS requires the subcutaneous insertion of small catheter containing a glucose sensor and a transmitter. The glucose sensor utilizes interstitial glucose to calculate blood glucose levels, and records and stores glucose measurements every five minutes for multiple days.

\section{Omron HJ-720 ITC Pedometer}

The Omron HJ-720 ITC pedometer (Omron, Lake Forest, IL) was worn in the pants pocket to document daily physical activity. This pedometer has been shown to be accurate when 
120 worn in this location in pregnant women (Connolly et al., 2011). Steps were averaged for days 121 two and four since 24-hour data were available for each of these days.

122

123

124

125

126

127

128

129

130

131

132

\section{Procedures}

Each participant began the study on Day 1 and concluded on Day 5. Days 2 and 4 were the experimental days, when the women either walked or remained sedentary. Day 3 did not require women to report to the clinic. On Day 5, the CGMS was removed and the participant returned her glucose logs and pedometer.

On Day 1, the CGMS was placed above the hip on the lower back (Medtronic Inc., 2004). On Days 2 and 4, the participants reported to the clinic after fasting at least two hours and were provided a prepackaged meal (60 grams $\mathrm{CHO}, 10$ grams protein, 7 grams fat; $400 \mathrm{Kcal})$. After eating, the participants sat for 30 minutes and glucose was assessed using the participants' personal glucometers. The participants were then randomly assigned to either 30 minutes of treadmill walking or 30 minutes of sitting.

\section{Walking Trial}

The participants walked for 30 minutes at $80.4 \mathrm{~m} \bullet \mathrm{min}^{-1}$ (3.3 METs), following physical activity recommendations (ACOG, 2015, Wolfe \& Davies, 2003). The speed of the treadmill was adjusted if the intensity of the activity was too high as determined by telemetry-measured heart rate (Davies et al., 2003) (out of recommended range for maternal age), RPE ( $\geq 14$ Borg Scale), or if the participant was uncomfortable walking at that pace. Following the walking bout, blood glucose was assessed. Fetal heart rate was assessed by Doppler (Huntleigh Dopplex FD3; Wales UK) before and within five minutes of completing the walking bout. The recommended fetal heart range is $110-160$ beats $\bullet \mathrm{min}^{-1}$ (ACOG, 2009). 


\section{Sedentary Trial}

During this trial, participants sat and talked with researchers for 30 minutes. Before and

144 after the sedentary trial, blood glucose and fetal heart rates were assessed.

\section{Statistical Analyses}

The primary outcome variables evaluated were postprandial (i.e., immediately prior to the

147 walking bout or sedentary bout) and post-treatment (i.e., immediately following the walking bout 148 or sedentary bout) glucose levels obtained from glucometers and continuous, 24-h CGMS blood 149 glucose measurements. Glucose and the area under the curve for glucose $\left(\mathrm{AUC}_{\mathrm{Glucose}}\right)$ from one 150 until six hours postprandial were calculated from CGMS output. Differences in glucose levels 151 and $\mathrm{AUC}_{\text {Glucose }}$ were analyzed over time using paired t-tests and a repeated-measures ANOVA.

152 An alpha level of $p<0.05$ was used to determine significance. In order to obtain a power of 0.80 , 153 a sample size of eight participants was needed. Post-hoc power analysis indicated an effect size 154 of 2.32, with a measured power of 0.999. All analyses were done using SPSS Statistics, Version $155 \quad 17.0$.

\section{Results}

There was one brief incidence of hypoglycemia $(\leq 3.9 \mathrm{mg} / \mathrm{dL})$ in a single subject that was resolved in the following hour. All measured fetal heart rates were within the normal range.

159 Thirty-minute postprandial glucose (pre-treatment) was not different between the walking and 160 sedentary trials. Immediate post-treatment glucose $(p<0.01)$ and two-hour postprandial glucose 161 levels $(p<0.05)$ were lower in the walking compared to the sedentary trial (Figure 1$)$. When all 162 glucose values for the 24-h periods following the trials were averaged, glucose measurements 163 were not significantly different $\left[5.1 \pm 0.2 \mathrm{mmol} \cdot \mathrm{L}^{-1}\right.$ (walking) vs. $5.2 \pm 0.5 \mathrm{mg} \bullet \mathrm{dL}^{-1}$ (sedentary)]. 
$164 \mathrm{AUC}_{\mathrm{Glucose}}$ was lower for the walking bout compared to the sedentary trial, for three hours after

165 the meal (Table 1). There was a trend towards differences in $\mathrm{AUC}_{\text {Glucose }}$ between the walking 166 and sedentary trials in hours 4-6 after the meal (Table 1).

Maternal heart rate averaged $134 \pm 12$ beats $\bullet \mathrm{min}^{-1}$ (walking) and $85 \pm 9$ beats $\bullet \mathrm{min}^{-1}$

168 (sedentary). Fetal heart rate averaged $147 \pm 7$ beats $\bullet \mathrm{min}^{-1}$ following the walking trial and $142 \pm 11$

169 beats $\bullet \mathrm{min}^{-1}$ after the sedentary trial. Pedometer step counts for the women averaged 7,111 $\pm 1,221$

170 steps on the walking day and 3,838+1,925 steps on the sedentary day. The women averaged

171 approximately 3,300 steps during the 30-minute walking trial, which accounts for this step

172 difference.

173 Discussion

This study utilized a bout of physical activity consistent with current guidelines and

175 investigated blood glucose changes in women with GDM after an acute bout of moderate-

176 intensity walking following a high $\mathrm{CHO} /$ low fat meal. The primary findings indicate that a 30 -

177 minute bout of moderate intensity walking resulted in lower postprandial glucose levels for two

178 hours after activity and better glucose control ( $\left.\mathrm{AUC}_{\text {glucose }}\right)$ for up to three hours postprandial on

179 the walking day compared to the sedentary day.

There is limited research regarding blood glucose changes after an acute bout of physical

181 activity. Avery and Walker (2001) looked at glucose levels under three conditions: resting,

182 cycling at a low intensity, and cycling at a moderate-intensity two hours after a meal. The

183 authors found that the women had more favorable blood glucose levels for up to 45 minutes after

184 exercise compared to the rest day only with the moderate intensity cycling (Avery \& Walker,

185 2001). Garcia-Paterson and colleagues (2001) compared blood glucose levels of women with 
186 GDM after the women completed a 60 -minute walk $\left(2.52 \mathrm{~km}^{\prime} \mathrm{hr}^{-1}\right)$ immediately following a 187 meal. Results showed a decreased postprandial glucose excursion for one hour after the meal

188 (Garcia-Patterson et al., 2001). The findings from these studies suggest that physical activity 189 after a meal has a greater impact on postprandial glucose levels in women with GDM, compared 190 to activity before a meal. In the current study, the women performed a bout of moderate 191 intensity walking 30 minutes after a meal, and the results showed better glucose control for up to 192 three hours postprandial. The duration of glucose control in the current study is greater than what was previously 194 reported. In addition to the timing of the activity, these results could also possibly be due to the 195 use of weight-bearing, moderate-intensity activity. This type and intensity of activity may 196 increase the magnitude of physical activity's effect on glucose control in women with GDM. 197 Avery and Walker utilized cycling (non-weight-bearing activity) at low and moderate intensities 198 and only found an effect with moderate intensity (2001). Garcia-Paterson et al. (2001) had their 199 participants walk at a low intensity. Although they utilized low intensity activity, the weight200 bearing nature may be responsible for the increased glucose control for a slightly longer duration 201 compared to Avery and Walker (Garcia-Patterson et al., 2001). Moderate-intensity, weight 202 bearing activity also leads to a greater caloric expenditure compared to non-weight-bearing, 203 moderate-intensity activity, which may enhance glucose uptake by the muscles. researchers and clinicians focus on postprandial glucose levels instead of fasting blood glucose.

207 Postprandial glucose levels may have a greater impact on disease-related complications and 208 adverse effects on individuals with diabetes. Higher peak postprandial glucose levels can 
209 potentially induce a hyperglycemic state; increasing the amount of glucose supplied to the fetus.

210 Although this has not been an area of focus in women with GDM, it is possible that higher

211 postprandial glucose levels, which tend to exceed fasting glucose levels, may have a greater

212 negative impact on the fetus compared to higher fasting glucose levels (Heine et al., 2004).

Study limitations include a small sample size and limited dietary control. However, there

214 was sufficient power to find significant differences in postprandial glucose levels. Detailed

215 dietary data were available for the meals provided on the study days, but no dietary data on the

216 remainder of the study days are available. A significant strength of this study was the use of the

217 CGMS, which allowed the researchers to monitor time-course changes in blood glucose values

218 that result from food intake and activity. Additionally, the activity prescribed during this study

219 aligned with current guidelines.

In conclusion, moderate-intensity walking had a positive impact on postprandial glucose

221 levels for up to two hours and better glucose control $\left(\mathrm{AUC}_{\mathrm{glucose}}\right)$ for up to three hours after the

222 meal. These findings suggest that women who participate in postprandial activity consistent with

223 the guidelines can reduce postprandial glucose levels and improve glucose profiles. Future

224 studies should investigate the thresholds for duration, intensity, and timing of activity, and

225 impact of chronic physical activity participation on postprandial glucose and glucose control in

226 women with GDM.

\section{Conflict of Interest Disclaimer}

228 The authors have no conflicts of interest to disclose regarding this manuscript. 
232 Connolly for assistance with the IRB application, Doree Gardner for manuscript editing, and

233 Dana Wolff and Brittney Wiseman for assistance with data collection. 


\section{References}

American College of Obstetrics and Gynecology. 2015. ACOG committee opinion: Physical Activity and Exercise during Pregnancy and the Postpartum Period. 2015. Number 650. PMID: 16319279.

American College of Obstetrics and Gynecology. 2009. ACOG Practice Bulletin No. 106: Intrapartum fetal heart rate monitoring: nomenclature, interpretation, and general management principles. (2009). Obstet. Gynecol. 114(1): 192-202. DOI: 10.1097/AOG.0b013e3181aef106. PMID: 19546798.

Artal, R. 2003. Exercise: the alternative therapeutic intervention for gestational diabetes. Clin. Obstet. Gynecol. 46(2): 479-487. PMID: 12808397.

Avery, M. D., \& Walker, A. J. 2001. Acute effect of exercise on blood glucose and insulin levels in women with gestational diabetes. J. Matern. Fetal. Med. 10(1): 52-58. PMID: 11332421.

Bung, P., Artal, R., Khodiguian, N., \& Kjos, S. 1991. Exercise in gestational diabetes. An optional therapeutic approach? Diabetes, 40 (Suppl 2): 182-185. PMID: 1748256.

Canadian Society for Exercise Physiology. 2002. Physical Activity Readiness Medical Examination for Pregnancy. Retrieved April 24, 2017, from http://www.csep.ca/cmfiles/publications/parq/parmed-xpreg.pdf

Chen, L., Mayo, R., Chatry, A., \& Hu, G. 2016. Gestational Diabetes Mellitus: Its Epidemiology and Implication beyond Pregnancy. Curr. Epidemiol. Rep. 3(1):1-11. doi:10.1007/s40471-016-0063-y.

Colberg, S.R., Sigal, R.J., Yardley, J.E., Riddell, M.C., Dunstan, D.W., Dempsey, P.C., Horton, 
E.S., Castorino, K. \& Tate, D.F. 2016. Physical Activity/Exercise and Diabetes: A Position Statement of the American Diabetes Association Diabetes Care. 39(11): 20652079. doi:10.2337/dc16-1728.

Connolly, C. P., Coe, D. P., Kendrick, J. M., Bassett, D. R., Jr., \& Thompson, D. L. 2011. Accuracy of physical activity monitors in pregnant women. Med. Sci. Sports. Exerc. 43(6): 1100-1105. doi: 10.1249/MSS.0b013e3182058883. PMID: 21085037.

Davenport, M. H., Mottola, M. F., McManus, R., \& Gratton, R. 2008. A walking intervention improves capillary glucose control in women with gestational diabetes mellitus: a pilot study. Appl. Physiol. Nutr. Metab. 33(3): 511-517. doi: 10.1139/H08-018. PMID: 18461104.

Davies, G. A., Wolfe, L. A., Mottola, M. F., MacKinnon, C., Society of Obstetricians \& Gynecologists of Canada. 2003. Joint SOGC/CSEP clinical practice guideline: exercise in pregnancy and the postpartum period. Can. J. Appl. Physiol. 28(3): 330-341. PMID: 12955862

Garcia-Patterson, A., Martin, E., Ubeda, J., Maria, M. A., de Leiva, A., \& Corcoy, R. 2001. Evaluation of light exercise in the treatment of gestational diabetes. Diabetes Care, 24(11): 2006-2007. PMID: 11679479.

Heine, R. J., Balkau, B., Ceriello, A., Del Prato, S., Horton, E. S., \& Taskinen, M. R. 2004. What does postprandial hyperglycaemia mean? Diabet. Med. 21(3): 208-213. PMID: 15008828.

Jovanovic-Peterson, L., Durak, E. P., \& Peterson, C. M. 1989. Randomized trial of diet versus diet plus cardiovascular conditioning on glucose levels in gestational diabetes. Am. J. Obstet. Gynecol. 161(2): 415-419. PMID: 2764059. 
281 Kerssen, A., de Valk, H. W., \& Visser, G. H. 2004. The Continuous Glucose Monitoring System

282

283

284

285

286

287

288

289

290

291

292

293

294

295

296

297

298

299

300

301

302

303 during pregnancy of women with type 1 diabetes mellitus: accuracy assessment. Diabetes Technol. Ther. 6(5): 645-651. doi: 10.1089/dia.2004.6.645. PMID: 15628818.

Kerssen, A., De Valk, H. W., \& Visser, G. H. 2005. Validation of the Continuous Glucose Monitoring System (CGMS) by the use of two CGMS simultaneously in pregnant women with type 1 diabetes mellitus. Diabetes Technol. Ther. 7(5): 699-706; discussion 707-699. doi: 10.1089/dia.2005.7.699. PMID: 16241871.

Lesser, K. B., Gruppuso, P. A., Terry, R. B., \& Carpenter, M. W. 1996. Exercise fails to improve postprandial glycemic excursion in women with gestational diabetes. J. Matern. Fetal Med. 5(4): 211-217. doi: 10.1002/(SICI)1520-6661(199607/08)5:4<211::AIDMFM9>3.0.CO;2-N. PMID: 8796796.

Manders, R. J., Van Dijk, J. W., \& van Loon, L. J. 2010. Low-intensity exercise reduces the prevalence of hyperglycemia in type 2 diabetes. Med Sci Sports Exerc. 42(2): 219-225. doi: 10.1249/MSS.0b013e3181b3b16d. PMID: 19927038.

Medtronic, Inc. 2004. Sof-Sensor - Glucose Sensor User Guide.

Public Health Agency of Canada. 2014. Maternal Diabetes in Canada. Ottawa, Ontario, Canada. Wolfe, L. A. and Davies, G. A. 2003. Canadian guidelines for exercise in pregnancy. Clin. Obstet. Gynecol. 46(2): 488-495. PMID: 12808398. 


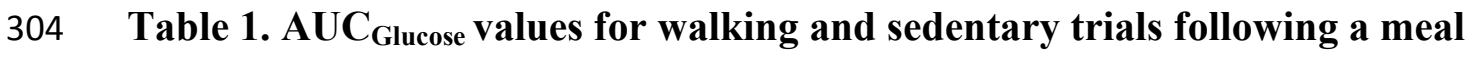

\begin{tabular}{lccc}
\hline Time after meal & $\mathbf{A U C}_{\text {Glucose }}$ Walking Trial & AUC $_{\text {Glucose }}$ Sedentary Trial & $p$ value \\
\hline One hour & $5.3 \pm 0.2$ & $6.5 \pm 0.7$ & 0.001 \\
Two hours & $10.2 \pm 0.5$ & $12.0 \pm 1.2$ & 0.003 \\
Three hours & $15.3 \pm 1.2$ & $17.2 \pm 1.7$ & 0.018 \\
Four hours & $20.5 \pm 1.9$ & $22.7 \pm 2.4$ & 0.058 \\
Five hours & $25.5 \pm 2.6$ & $28.3 \pm 3.2$ & 0.057 \\
Six hours & $30.3 \pm 3.3$ & $33.8 \pm 4.0$ & 0.053 \\
\hline
\end{tabular}

307 Figure 1. Postprandial blood glucose levels following walking and sedentary trials. The 308 solid line represents glucose levels during walking trial and the dashed line represents 309 glucose levels during the sedentary trial. 
Applied Physiology, Nutrition, and Metabolism

Page 16 of 16

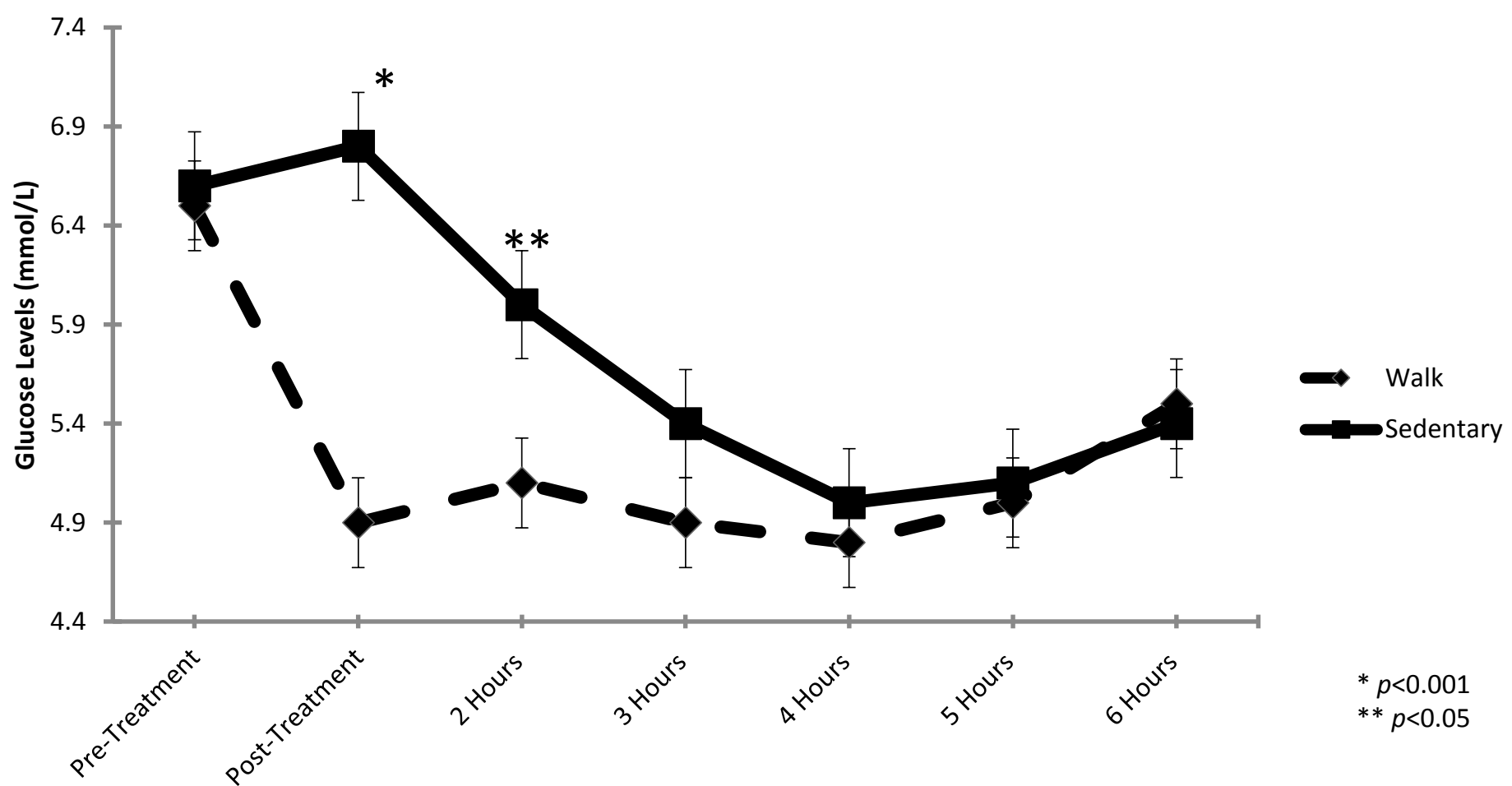

https://mc06.manuscriptcentral.com/apnm-pubs 